\title{
Mesure de l'allergénicité des denrées alimentaires - développement de modèles
}

Oléagineux, Corps Gras, Lipides. Volume 10, Numéro 1, 23-4, Janvier - Février 2003, Protéines et lipides végétaux : interactions nutritionnelles et fonctionnelles

Auteur(s) : Daniel TOMÉ, Institut National Agronomique Paris-Grignon, 16, rue Claude Bernard, 75231 Paris cedex 05.

Résumé : La sensibilisation de l'organisme par des allergènes d'origine alimentaire s'exprime par I'apparition d'immunoglobulines spécifiques (IgE). Ces allergies sont provoquée dans la quasi totalité des cas par des protéines. De nombreux aliments sont susceptibles de provoquer des allergies chez certains sujets sensibles. On trouve ainsi des allergies aux Drupacée (approximativement $16 \%$ de la fréquences des allergies alimentaires), aux Ombellifères (11\%), à l'œuf (8\%), aux Légumineuses (3 $\%)$, aux crustacés (8\%), au poisson (7\%), à l'avocat et au kiwi (5\%), au lait (5\%), à la farine de blé (4 $\%)$, à la banane (3\%). Parmi les allergies alimentaires, l'allergie à l'arachide est particulièrement fréquente. Dans le cas des réactions croisées, l'organisme est sensibilisé par un premier contact avec un allergène distinct de celui qui va induire les réactions immunitaires excessives et aberrantes. Afin d'évaluer le potentiel allergénique de protéines alimentaires il est nécessaire de disposer de tests permettant de contrôler les matières premières et d'évaluer les moyens technologiques permettant de réduire l'allergénicité de certains produits. Des modèles d'allergie à l'arachide par sensibilisation orale, nasale ou intrapéritonéale sont développés chez la souris. Les données disponibles indiquent qu'il est possible de reproduire certains aspects de la sensibilisation et des réactions croisées observées chez l'homme. Ces modèles de sensibilisation permettent d'évaluer le potentiel allergénique de protéines alimentaires.

Mots-clés : antigène et allergène alimentaire, réactions croisées, sensibilisation, arachide, modèle animal.

\section{ARTICLE}

Les réactions allergiques sont des réactions immunologiques excessives et aberrantes de l' organisme en réponse à un antigène particulier ou allergène. $L^{\prime}$ ingestion de protéines alimentaires se traduit généralement par une réponse immune non allergique (phénomène de tolérance). La transition vers $l^{\prime}$ allergie alimentaire reste mal comprise. Les allergènes alimentaires sont $d^{\prime}$ origines diverses et tous les aliments sont potentiellement des sources $d$ 'allergènes. Un nombre limité $d$ ' aliment est cependant responsable de plus de $90 \%$ des allergies alimentaires (exemple de l' arachide).

La sensibilisation de l' organisme par des allergènes d' origine alimentaire s' exprime par l'apparition d'immunoglobulines spécifiques (IgE). La prévalence de ce type d'allergie reste mal définie mais est probablement proche de $1 \%$ de la population. Ces allergies sont provoquée dans la quasi totalité des cas par des protéines et souvent des glycoprotéines hydrosolubles à point isolélectrique acide et de 
masse moléculaire entre 10,000 et 70,000 daltons. Les conditions de l' allergénicité sont de plusieurs ordres. Le terrain génétique (ou atopie) prédispose certains individus à la synthèse d' IgE spécifiques. Les caractéristiques des protéines allergéniques interviennent aussi en leur conférant une résistance à la dénaturation thermique (arachide, soja, crevette, poisson, ovomucoïde), une résistance à la protéolyse (poisson, ovomucoïde, moutarde, inhibiteurs de protéases), une aptitude particulière à $l^{\prime}$ accès à la muqueuse intestinale et aux cellules de l' immunité, une aptitude à l' endocytose favorisant le transfert et la présentation aux lymphocytes $T$.

Les allergènes alimentaires sont donc des antigènes susceptibles de provoquer une réponse immunitaire particulière dite "allergique" liée à la synthèse d' IgE spécifiques. Les allergènes dits "majeurs" provoquent une réaction chez $50 \%$ des patients. Les propriétés allergéniques sont liées à une ou des portions limitées de la protéines, les épitopes. Pour être immunogène I' allergène doit contenir des épitopes vis à vis des lymphocytes B et $T$ et des agrétopes pour la présentation initiale par les cellules présentant l' antigène. Un épitope est un peptide comportant le plus souvent de 8 à12 acides aminés. Les acides aminés adjacents sont important pour maintenir la configuration active. Le déterminant antigénique comprend donc environ 20 acides aminés. Les épitopes sont généralement situés en surface de la protéine dans des zones flexibles et hydrophiles. On distingue en outre les épitopes conformationels, détruits par perte de la structure tertiaire de la protéine, et les épitopes séquentiels, dépendants de l' enchaînement des acides aminés et détruits par coupure de la séquence des acides aminés.

De nombreux aliments sont susceptibles de provoquer des allergies chez certains sujets sensibles. On trouve ainsi des allergies aux Drupacée (approximativement $16 \%$ de la fréquences des allergies alimentaires), aux Ombellifères (11\%), à l'œuf (8\%), aux Légumineuses (3\%), aux crustacés (8\%), au poisson (7\%), à l'avocat et au kiwi (5\%), au lait (5\%), à la farine de blé (4\%), à la banane (3\%). Parmi les allergies alimentaires, I' allergie à l' arachide est particulièrement fréquente. Elle touche de l'ordre de $1 \%$ de la population américaine par exemple et ne diminue pas avec l' âge. Elle provoque une réaction allergique sévère et potentiellement fatale avec une forte prévalence de symptômes suite à un contact minimum. En provocation oral, $25 \%$ des sujets allergiques répondent à une dose inférieure à $100 \mathrm{mg}$ et $88 \%$ à une dose inférieure à $1 \mathrm{~g}$. La présence de l' arachide dans de nombreux ingrédients et aliments pose donc un réel problème de contrôle.

Un problème particulier et délicat concerne les réactions allergiques croisées. Dans le cas des réactions croisées, $\mathrm{l}^{\prime}$ organisme est sensibilisé par un premier contact avec un allergène distinct de celui qui va induire les réactions immunitaires excessives et aberrantes. Cela est généralement lié à de fortes homologies de structure de certains composant dits "panallergènes" entre les deux espèces. Différents cas d'allergie croisées doivent être envisagées: les allergies croisées poliniques/alimentaires et les allergies croisées alimentaires/alimentaires. L' importance réelle de ces phénomènes reste mal connue mais représente une question majeure pour le développement de sources nouvelles de protéines alimentaires. Des réactions allergiques croisées ont ainsi été soupçonnées dans la famille des Légumineuses. Le problème le plus souvent cité est la réaction croisée entre l'arachide comme allergène primaire de sensibilisation et d'autres légumineuses comme allergène réagissant secondairement à une sensibilisation à l'archide. II a cependant été noté 
que l'existence de réaction croisée in vitro vis-à-vis des IgE n'était pas corrélée de façon directe avec des symptômes cliniques d'allergie obtenus par tests de provocation.

Afin d'évaluer le potentiel allergénique de protéines alimentaires il est nécessaire de disposer de tests permettant de contrôler les matières premières et $d^{\prime}$ évaluer les moyens technologiques permettant de réduire l' allergénicité de certains produits. Il est aussi important de pouvoir étudier la réalité des allergies croisées entre les différentes légumineuses. Les études chez l'homme sont limitée. La provocation orale est dangereuse et les mécanismes ne peuvent être étudiés de façon approfondie chez l' homme. II est donc nécessaire de diposer de modèles animaux pour analyser les mécanismes de l' allergie alimentaire et comprendre et prévenir les réactions allergiques directes et croisées. Un modèle de choix est celui de la souris allergiques à l' arachide. Une réaction allergique peut ainsi être provoquée chez la souris par immunisation orale, nasale ou intrapéritonéale par un extrait $d^{\prime}$ arachide en présence $d^{\prime}$ un adjuvant (alun, toxine cholérique, ...). La sensibilisation par voie respiratoire est un des mécanismes possible à l' origine $d^{\prime}$ 'effets $d^{\prime}$ allergies alimentaires. Les symptômes allergiques sont plus facilement induits par immunisation nasale que par la voie orale. L'étude des modèles de sensibilisation montre qu'une sensibilisation intrapéritonéale à l' arachide conduit à des réponses variables selon les souches de souris (C3H, CBA, SJL, BALB/c). Ainsi, si toutes les souches de souris développent des IgG anti-arachide, concernant les IgE les meilleures réponses (niveau des réponses et nombre $d^{\prime}$ animaux positifs) sont obtebues avec la souche $\mathrm{CBA}\left(\mathrm{H}-2^{\mathrm{k}}\right)$ suivi par la souche $B A L B / c\left(H-2^{d}\right)$. En outre une grande variabilité individuelle est observée dans les niveaux de réponse au sein $d^{\prime}$ une même souche de souris. Les souris d' haplotype $\mathrm{H}-2 \mathrm{~s}$ (SIL) (C57BL/6) immunisées avec de l' arachide et de la toxine cholérique ne développent pas de réponse IgE. Enfin, l'étude des réactions croisées de sérum d'animaux sensibilisées à l'arachide avec d'autres légumineuses ne montrent pas de différence significative entre les différentes légumineuses.

\section{CONCLUSION}

En conclusion la prédiction et la prévention de l' allergie alimentaire sont des contraintes de santé publique. L' exclusion des composants alimentaires responsables des réactions allergiques reste le moyen le plus sûr de prévenir ces réactions indésirables. Un marché est ouvert pour le développement d' aliments "hypo-allergéniques" destinés à des groupes à risque ou à l' ensemble de la population. Les développements sont fortement conditionnés par une meilleure connaissance des bases objectives de l' allergénicité. L' expérimentation étant délicate chez l' homme. Des modèles d' allergie à l' arachide par sensibilisation orale, nasale ou intrapéritonéale sont développés chez la souris. Les données disponibles indiquent qu' il est possible de reproduire certains aspects de la sensibilisation et des réactions croisées observées chez l' homme. Ces modèles de sensibilisation permettent d' évaluer le potentiel allergénique de protéines alimentaires, de disposer de tests permettant de contrôler les matières premières et $d^{\prime}$ évaluer les moyens technologiques permettant de réduire l' allergénicité de certains produits, d' étudier la réalité des allergies croisées entre les différentes protéines alimentaires. 


\section{REFERENCES}

1 - Sampson HA. Immediate hypersensitivity reactions to foods : blinded food challenges in children with atopic dermatitis. Ann Allergy 1986 Sep ; 57 (3) : 209-12.

2 - Sampson HA, McCaskill CC. Food hypersensitivity and atopic dermatitis : evaluation of 113 patients. J Pediatr 1985 Nov ; 107 (5) : 669-75.

3 - Romano C, Ferrara A, Falagiani P. A case of allergy to globe artichoke and other clinical cases of rare food allergy. J Investig Allergol Clin Immunol 2000 Mar-Apr ; 10 (2) : 102-4.

4 - Parisot L, Aparicio C, Moneret-Vautrin DA, Guerin L. Allergy to lupine flour. Allergy 2001 Sep ; 56 (9) : 918-9.

5 - Hefle SL, Helm RM, Burks AW, Bush RK. Comparison of commercial peanut skin test extracts. J Allergy Clin Immunol 1995 Apr ; 95 (4) : 837-42.

6 - Bock SA, Atkins FM. The natural history of peanut allergy. J Allergy Clin Immunol 1989 May ; 83 (5) : 900-4.

7 - Bock SA, Atkins FM. Patterns of food hypersensitivity during sixteen years of double-blind, placebo-controlled food challenges. J Pediatr 1990 Oct ; 117 (4) : 561-7.

8 - Bernhisel-Broadbent J, Sampson HA. Cross-allergenicity in the legume botanical family in children with food hypersensitivity. J Allergy Clin Immunol 1989 Feb ; 83 (2 Pt 1) : 435-40. 